\title{
COMUNICAÇÃO BOCA A BOCA COMO INSTRUMENTO DE MARKETING PARA MICRO E PEQUENAS EMPRESAS
}

\section{WORD OF MOUTH COMMUNICATION AS AN INSTRUMENT OF MARKETING FOR MICRO AND SMALL ENTERPRISES}

\author{
MARIANA TORRES UCHOA \\ Universidade de São Paulo (USP) \\ Mestre em Administração pela Universidade Federal de Goiás \\ Orcid: http://orcid.org/0000-0001-5134-6659 \\ E-mail: marianauchoa@outlook.com \\ RICARDO LIMONGI FRANÇA COELHO \\ Universidade Federal de Goiás (UFG) \\ Doutor em Administração pela EAESP/FGV \\ Orcid: http://orcid.org/0000-0003-3231-7515 \\ E-mail: ricardolimongi@ufg.br

\section{ANDRÉ LUIZ BARBOZA DA SILVA} \\ Universidade Federal de Goiás (UFG) \\ Doutor em Administração pela EAESP/FGV \\ Orcid: http://orcid.org/0000-0003-3276-5019 \\ E-mail:andre_luiz_b_silva@hotmail.com
}

Avenida Professor Luciano Gualberto, 908 - Butantã, São Paulo/SP, CEP: 05508-010

Submissão: 03/05/2020. Revisão: 24/03/2021. Aceite: 02/07/2021. Publicação: 28/07/2021. DOI: http://dx.doi.org/10.22277/rgo.v14i3.5442

\section{RESUMO}

As Micro e Pequenas Empresas (MPEs) possuem relevância para a economia do país e limitações no uso dos recursos do marketing. Como forma de contribuir com essa discussão, este estudo avalia o marketing empreendedor com ênfase no marketing boca a boca, principal meio de divulgação das MPEs. Assim, o objetivo é analisar as variáveis que impactam o boca a boca nas MPEs: qualidade do alimento, do serviço, da atmosfera e do preço quando um consumidor é motivado pela preocupação com os outros a expressar seus sentimentos. Com uma amostra com 145 consumidores foi utilizada a análise de equações estruturais e como resultado foi identificado que a atmosfera influencia o boca a boca realizado pela preocupação com os outros, e a qualidade do serviço influencia o boca a boca a partir da motivação de expressar os sentimentos e ajudar o restaurante. Estes resultados contribuem para que gestores possam delimitar suas estratégias, visando o marketing boca a boca.

Palavras-chave: Boca a boca. Marketing empreendedor. Micro e pequenas empresas (MPEs). Restaurante. 


\begin{abstract}
Micro and Small Enterprises (MSEs) have relevance to the country's economy and limitations in using marketing resources. To overcome this limitation, this study evaluates entrepreneurial marketing with an emphasis on the word of mouth marketing, the main means of disseminating MSEs. Thus, the objective is to analyze the variables that impact word of mouth in MSEs: quality of food, service, atmosphere, and price when a consumer is motivated by the concern for others to express their feelings. With a sample of 145 consumers, the analysis of structural equations was used, and as a result, it was identified that the atmosphere influences word of mouth through concern for others, and the quality of service influences word of mouth based on the motivation to express feelings and help the restaurant. These results are an important practical implication for managers to define their strategies for word-of-mouth marketing.
\end{abstract}

Keywords: Word of mouth. Entrepreneurial marketing. Micro and small businesses (SMEs). Restaurants.

\title{
1 INTRODUÇÃo
}

O segmento de restaurante e alimentação vem passando por mudanças no comportamento de consumo, em que o estilo de vida, a falta de tempo e os hábitos influenciaram na forma de alimentação, aumentando o consumo fora de casa (ABRASEL, 2019; BAHAUDDIN; WEI; MANTIHAL, 2020). De acordo com a Associação Brasileira de Bares e Restaurantes (ABRASEL), do orçamento destinado a alimentação, as famílias gastam em média $32,8 \%$ com o consumo fora de casa (ABRASEL, 2019) e durante a pandemia do Covid-19, apesar das restrições, os restaurantes se aperfeiçoaram, aderiram a modalidade delivery e estão entregando experiências de consumo satisfatórias para os consumidores (ABRASEL, 2021). Um destaque e líder de consumo são as hamburguerias (ABRASEL, 2021).

Com consumidores cada vez mais exigentes a busca por inovações para atrair e mantêlo devem ser constantes (KAEWMAHAPHINYO; NUANGJAMNONG; DOWPISET, 2020; ŽIVADINOVIĆ, 2020). Além disso, com a variedade e número de concorrentes do setor, as empresas devem ser capazes de criar estratégias de sobrevivência atendendo a demanda dos diferentes públicos que consomem o produto (SOLUNOĞLU, 2020). Neste sentido, uma importante fonte de vantagem competitiva para as lanchonetes é a experiência do cliente (MATHAYOMCHAN; TAECHARUNGROJ, 2020).

A experiência e qualidade percebida pelos consumidores no segmento de restaurante possuem um forte impacto na satisfação e comportamento pós-consumo (BAKER; CROMPTON, 2000; JEONG; JANG, 2011). Esta satisfação desempenha um papel fundamental na sobrevivência de uma empresa, uma vez que pode ter um impacto positivo no comportamento subsequente dos clientes, por exemplo, por meio da repetição da compra, da fidelização e da comunicação pessoal feita por um cliente (IGLESIAS; GUILLÉN, 2004). Ao estabelecer uma gestão eficaz dos serviços, as empresas podem potencializar o compartilhamento positivo por meio do boca a boca, conhecido na literatura como word of mouth (WOM) e definida como a comunicação que envolve consumidores que compartilham sua avaliação após a experiência de serviço (CASIDY; SHIN, 2015).

Os consumidores apreciam o conhecimento informal sobre produtos/serviços e marcas na comunicação do boca a boca, porque este formato é percebido como mais confiável do que as comunicações formais realizadas pelas empresas (BROWN et al., 2005; ZHANG; MA; 
CARTWRIGHT, 2013; JUNG; SEOCK, 2017). Além de mais confiável, a estratégia do boca a boca pode gerar benefícios positivos para as empresas, como maior incentivo à escolha da marca (EAST; HAMMOND; LOMAX, 2008) e aumento da captação de novos clientes gerados pelas recomendações (KIM; NG; KIM, 2009). No entanto, uma vez que os clientes ficam insatisfeitos, frequentemente, engajam-se no boca a boca negativo, gerando impacto para marca e redução da fidelidade com a empresa (MILLER; CRAIGHEAD; KARWAN, 2000).

Diversas pesquisas ressaltam a importância e o impacto do boca a boca, como por exemplo, com ênfase na mediação da confiança na relação entre satisfação do cliente e boca a boca (LOUREIRO; CAVALLERO; MIRANDA, 2018), sobre o impacto do boca a boca na recuperação de serviços (NOONE, 2012; CASIDY; SHIN, 2015; JUNG; SEOCK, 2017), na influência de moderação negativa e positiva do boca-a boca nos resultados da comunidade da marca (RELLING et al., 2016), no impacto das avaliações boca a boca online dos consumidores no processo da compra (EAST; HAMMOND; LOMAX, 2008; BAMBAUER-SACHSE; MANGOLD, 2011; EAST; HAMMOND; ZHANG; MA; CARTWRIGHT, 2013), no engajamento positivo no ambiente online (JEONG; JANG, 2011), sobre a influência da atmosfera dos serviços na satisfação e no boca a boca (KIM; NG; KIM, 2009) e em relação aos principais antecedentes do boca a boca positivo (BROWN et al., 2005).

Os serviços, por serem intangíveis, não permitem a avaliação dos consumidores antes do consumo, portanto, a compra de serviços traz maior risco, pois os clientes ficam mais dependentes do boca a boca. Assim, uma experiência positiva do consumidor pode ser criada por meio de situações ou práticas na loja, como por exemplo: o serviço de atendimento, a atmosfera (ambiente) da loja, qualidade dos produtos oferecidos (MITCHELL et al., 2015; BAHAUDDIN; WEI; MANTIHAL, 2020; MATHAYOMCHAN; TAECHARUNGROJ, 2020) e preço (NAMIM, 2017, BAHAUDDIN; WEI; MANTIHAL, 2020; MATHAYOMCHAN; TAECHARUNGROJ, 2020) que acarretam um boca a boca positivo (MITCHELL et al., 2015). Dessa forma, essas variáveis serão analisadas no estudo.

Diante de uma realidade com restrição de recursos as micro e pequenas empresas (MPE) tem o marketing boca a boca como uma importante ferramenta de apoio de divulgação (IFIE, 2018), no entanto, pesquisas com ênfase nas MPEs no segmento de serviços sob a perspectiva do boca a boca ainda carecem de maior atenção. Para Jeong e Jang (2011), apesar do impacto significativo do boca a boca no ambiente virtual nas empresas de hospitalidades, especialmente no segmento de restaurantes, poucas pesquisas foram feitas para investigar o boca a boca neste campo.

Ampliar o entendimento sobre esse tema é importante pois as MPEs têm importância significativa na economia brasileira: representam $98,5 \%$ das empresas privadas, correspondem a $27 \%$ do PIB nacional e são responsáveis por $54 \%$ dos empregos com carteira assinada (SEBRAE, 2018). Além disso, o segmento de restaurantes e lanchonetes ganha destaque entre as MPEs, sendo uma das atividades mais frequentes, representando $5,1 \%$ das microempresas e $4,2 \%$ das pequenas empresas (SEBRAE, 2018).

Sendo assim, com este estudo é possível contribuir com os gestores no processo de reestruturar seus restaurantes por meio de estratégias mercadológicas para aumentar a intenção de compra, reduzir os custos com a captação de novos clientes e direcionar os seus esforços promocionais. Neste sentido, o principal objetivo desta pesquisa é analisar as variáveis que impactam a comunicação boca a boca nas micro e pequenas empresas no segmento de restaurantes. Este estudo contribui para a literatura de micro e pequenas empresas por desenvolver relações e entender o que de fato influencia um melhor 
desempenho das empresas, assim como sugerido por Omar e Fraser (2011), que argumentam que a teoria de PME ainda demanda desenvolvimento.

$\mathrm{O}$ artigo está estruturado em seis partes além desta introdução. As três partes iniciais apresentam respectivamente, uma revisão sobre o boca a boca e suas motivações, experiência no ambiente de restaurantes e a relação entre o boca a boca e a experiência positiva em restaurante. Posteriormente é apresentada a metodologia aplicada nesta pesquisa, seguida dos resultados obtidos na análise e, por fim, as considerações finais do trabalho.

\section{REVISÃO TEÓRICA}

A revisão teórica irá abordar, inicialmente, a dinâmica da comunicação boca a boca no contexto de micro e pequenas empresas. Posteriormente, as hipóteses serão apresentadas a partir das variáveis de interesse do estudo, qualidade de produto, serviço, atmosfera e preço.

\subsection{BREVE DISCUSSÃO ACERCA DA DINÂMICA DA COMUNICAÇÃO BOCA A BOCA}

O boca a boca é um conselho informal passado entre consumidores e representa uma influência relevante no comportamento do consumidor (EAST; HAMMOND; LOMAX, 2008; IFIE, 2018), podendo ser feito de forma positiva ou negativa (NOONE, 2012). O boca a boca ocorre entre um comunicador não relacionado com a empresa e um receptor, acerca de determinado produto ou serviço oferecido (ARNDT, 1967). Harrison-Walker (2001), complementa ao descrever como sendo uma comunicação informal entre duas pessoas, sobre a sua percepção da marca, produto, serviço e qualidade, sendo a informação que mais influência na decisão do consumidor por refletir a opinião de alguém que realmente já conhece o produto.

A comunicação boca a boca pode motivar diretamente as decisões de consumo e lealdade, pois são baseados na confiança e engajamento o que gera uma maior credibilidade para a opinião dada (JUNG; SEOCK, 2017; IFIE, 2018) e, portanto, pesquisadores avaliaram os seus efeitos sobre o comportamento do consumidor (JUNG; SEOCK, 2017). A comunicação positiva do boca a boca é divulgada a partir de encontros de serviços satisfatórios, enquanto a comunicação negativa geralmente segue encontros insatisfatórios (SUSSKIND, 2002). Os consumidores satisfeitos e insatisfeitos tendem a espalhar comentários positivos e negativos, respectivamente, sobre os itens que compraram e usaram (COLLIER; BIENSTOCK, 2006). De qualquer forma, o boca a boca, sendo positivo ou negativo, pode afetar a decisão de compra dos potenciais consumidores.

Sendo assim, este tipo de marketing está se tornando um ponto crítico em pesquisas sobre recuperação de serviços. Conforme Kau e Loh (2006), um consumidor insatisfeito que encontra uma falha de serviço, pode se tornar altamente envolvido em boca a boca negativo sobre a empresa prestadora deste serviço. Desta forma, quando o consumidor assume suas intenções e realiza a comunicação, seja positiva ou negativa, pode influenciar nas vendas e apresentar vantagens ou desvantagens mercadológicas para as empresas. O boca a boca possui custos consideravelmente mais baixos e com maior propagação (CHEVALIER; MAYZLIN, 2006). Dessa maneira, as empresas precisam garantir a satisfação e a confiança dos consumidores, já que essas variáveis têm uma associação positiva com a retenção e o endosso positivo na comunicação (RANAWEERA; PRABHU, 2003). Assim, o marketing boca a boca é um dos elementos que podem ser utilizados pelos pequenos empresários no sentido de inovação, afinal, é a partir da rede de relacionamentos que as opiniões começam a gerar ideias 
(STOKES, 2000; MITCHELL et al., 2015). É por meio das experiências que os consumidores das redes de relacionamentos possuem que os empreendedores conseguem captar as opiniões, melhorar os principais déficits internos, e assim, mobilizar cada vez mais pessoas para consumirem os produtos e serviços (MITCHELL et al., 2015).

\subsection{DELINEAMENTO DAS HIPÓTESES SOBRE QUALIDADE DE PRODUTO, SERVIÇO, ATMOSFERA E PREÇO}

O consumidor não consegue avaliar a prestação de serviços de restaurantes antes da experiência do consumo devido às questões intangíveis envolvidas no processo. Neste contexto, o consumidor pode sofrer maior influência do marketing boca a boca com empresas relacionadas à hospitalidade. Apesar do impacto significativo do boca a boca nessas empresas, especialmente no segmento de restaurantes, poucas pesquisas foram feitas para investigar tal efeito (JEONG; JANG, 2011; IFIE, 2018), em especial, para analisar as estratégias de marketing das PMEs, como por exemplo o marketing boca a boca (MITCHELL et al., 2015).

As expectativas são importantes porque quando são insatisfeitas criam clientes descontentes e podem espalhar o boca a boca negativo (KIM; NG; KIM, 2009). Os clientes de restaurantes que tiveram uma experiência de jantar agradável, provavelmente, seriam motivados a realizar o boca a boca positivo, ou seja, desempenharia um papel importante no aumento das intenções de compra dos clientes, criando uma imagem favorável da empresa e sua marca, e ainda, reduziriam as despesas promocionais (JEONG; JANG, 2011). Para os autores, quando os consumidores estão motivados pelo boca a boca é possível que se sintam preocupados com as outras pessoas e, assim, expressem sentimentos positivos e ajudem a empresa.

O "sentir-se preocupado" diz respeito a impedir que outras pessoas adquiram um produto ou serviço ruim, por isso, o boca a boca sofre forte influência dos consumidores, devido a intangibilidade dos serviços prestados nos restaurantes. $\mathrm{O}$ "expressar sentimentos positivos" é desencadeado por uma experiência de consumo positiva ou negativa do restaurante, em que o cliente tem um forte desejo de compartilhar a alegria ou decepção da experiência com outras pessoas. Por fim, "ajudar a empresa" é o mesmo motivo de preocupação para os outros: o altruísmo ou o desejo geral de ajudar os outros, neste caso, o restaurante.

Desta forma, as empresas precisam monitorar se as expectativas foram atendidas e quando os empreendedores do segmento de restaurantes compreendem a motivação do boca a boca, é possível elevar a intenção de compra dos consumidores (JEONG; JANG, 2011). Consequentemente, torna-se relevante compreender quais as variáveis podem influenciar neste processo. Pezenka e Weismayer (2020) identificaram que a qualidade do serviço, do próprio alimento, a atmosfera do restaurante e o preço foram os fatores mais utilizados na literatura e são os mais importantes para o tema, conforme apresentado no Quadro 1.

Em relação as avaliações dos clientes sobre a qualidade do serviço, o resultado é uma comparação das expectativas do serviço com o desempenho real (ZEITHAML; BERRY; PARASURAMAN, 1993; USLU 2020). Cabe ressaltar que as opiniões dos consumidores em restaurantes são construídas baseadas em vivências anteriores (USLU, 2020). Então, quando os clientes e experimentam o serviço de um restaurante, é avaliado se a experiência do restaurante foi boa ou não, ou seja, as percepções do consumidor e a qualidade do serviço podem ser altamente dependentes do desempenho da empresa durante a entrega (JEONG; JANG, 2011; USLU, 2020). Assim, em restaurantes, a qualidade percebida pelos clientes é revelada como um aspecto importante ao gerar satisfação (IGLESIAS; GUILLÉN, 2004).

RGO - Revista Gestão Organizacional, Chapecó, v. 14, n. 3, p. 175-193, set./dez. 2021. 
Mariana Torres Uchoa, Ricardo Limongi França Coelho e André Luiz Barboza da Silva

Quadro 1 - Estudos que analisaram os diferentes fatores que influencia na experiência de consumo

\begin{tabular}{|l|l|}
\hline & \\
\hline Erkmen (2019) & Aspecto cultural da comida, serviço \\
\hline Gan et al. (2017) & Comida, serviço, atmosfera e preço \\
\hline Hyun (2010) & Comida, serviço e preço \\
\hline Jacobsen and Haukeland (2002) & Comida, serviço, atmosfera, localização e preço \\
\hline Jeong and Jang (2011) & Comida, serviço e atmosfera \\
\hline Liu and Jang (2009) & Comida, serviço, atmosfera e preço \\
\hline Namkung and Jang (2008) & Comida, serviço, limpeza e preço \\
\hline Pantelidis (2010) & Comida, serviço e atmosfera \\
\hline Parsa et al. (2012) & Comida, serviço, atmosfera, menu e preço \\
\hline Ryu and Han (2010) & Comida, serviço e atmosfera \\
\hline Vu et al. (2019) & Comida, serviço e atmosfera \\
\hline Wall and Berry (2007) & Atmosfera, serviço e preço \\
\hline Yan et al. (2015) & Comida, serviço e atmosfera \\
\hline Zhang et al. (2014) & Comida, serviço, atmosfera, menu e valor \\
\hline
\end{tabular}

Fonte: adaptado de Pezenka e Weismayer (2020).

Neste sentido, no que tange a experiência em relação a qualidade do serviço uma hipótese é criada:

H1a: A qualidade do serviço influencia positivamente a preocupação com os outros. $\mathrm{H} 1 \mathrm{~b}$ : A qualidade do serviço influencia positivamente a expressar sentimentos. H1c: A qualidade do serviço influencia positivamente a ajudar o restaurante.

Dentre as variáveis que influenciam o boca a boca, a comida é um componente fundamental da experiência do restaurante e não há dúvida do impacto na satisfação do consumidor e do retorno (NAMKUNG; JANG, 2007). Os restaurantes possuem o desafio em fornecer aos seus consumidores alimentos de qualidade e superiores aos seus concorrentes, sendo um fator que influencia a fidelidade do cliente na escolha do restaurante (CLARK; KAMINSKI; RINK, 1992).

A qualidade da comida é percebida pelo aspecto sensorial (BAHAUDDIN; WEl; MANTIHAL, 2020), ou seja, é uma indicação sobre a excelência dos ingredientes e das refeições oferecidas, que inclui a limpeza, o frescor, a salubridade, bem como a variedade e apresentação dos alimentos (NAMIN, 2017; KONUK, 2019), aroma, sabor, textura e temperatura; e são esses aspectos que diferenciam a percepção do consumidor no quesito qualidade (BAHAUDDIN; WEI; MANTIHAL, 2020). Sendo que a variedade e a qualidade dos alimentos oferecidos estão entre os elementos mais importantes na satisfação do cliente no segmento de restaurantes (LAW; HUI; ZHAO, 2004; JEONG; JANG, 2011) e, portanto, nas intenções comportamentais pós-jantar para formação do boca a boca positivo ou negativo (JEONG; JANG, 2011).

De acordo com Mathayomchan e Taecharungroj (2020) e Pezenka e Weismayer (2020), a qualidade da comida foi considerada o aspecto mais importante que afeta a experiência do cliente. Entretanto, na percepção dos consumidores esse quesito tem sido amplamente negligenciado pelos estabelecimentos (ZHANG et al., 2021) e contribui na proposição da segunda hipótese da pesquisa:

H2a: A qualidade do produto em um restaurante (comida) influencia positivamente a preocupação com os outros.

$\mathrm{H} 2 \mathrm{~b}$ : A qualidade do produto em um restaurante (comida) influencia positivamente a expressão de sentimentos. 
H2c: A qualidade do produto em um restaurante (comida) influencia positivamente a ajudar o restaurante.

Outra variável relevante para os restaurantes é a atmosfera que é formada pela estrutura oferecida pelo restaurante como: decoração, luz, limpeza, música (USLU, 2020; ŽIVADINOVIĆ, 2020) e cores (ŽIVADINOVIĆ, 2020). Desta forma, a percepção da atmosfera pode aumentar ou reduzir o efeito da qualidade percebida na satisfação e em comportamentos positivos no pós-consumo (HA; JANG, 2010), podendo influenciar no boca a boca dos consumidores (JEONG; JANG, 2011). Os restaurantes devem também atentar-se que - ambiente é percebido pelos consumidores por meio dos cinco sentidos e, consequentemente, os estímulos sensoriais na atmosfera influenciam na satisfação e na intenção de compra (HEUNG; GU, 2012; ŽIVADINOVIĆ, 2020). Assim, a percepção do ambiente envolve emoções e influencia no consumo e também no tempo de permanência do cliente no restaurante/lanchonete (ŽIVADINOVIĆ, 2020). Consequentemente, se um cliente tem uma alta percepção do ambiente, sua expectativa quanto ao serviço e qualidade dos alimentos podem também ser altas e, em contrapartida, quando o cliente percebe uma atmosfera normal, onde as expectativas do consumidor não são superadas, ocorre uma menor satisfação do cliente e menores comportamentos de fidelidade (HA; JANG, 2010). Dessa forma, a terceira hipótese desta pesquisa é:

H3a: A atmosfera influencia positivamente a preocupação com os outros.

H3b: A atmosfera influencia positivamente a expressão de sentimentos.

H3c: A atmosfera influencia positivamente a ajudar o restaurante.

Para obter a satisfação do cliente e para que se tornem um recurso de comunicação boca a boca eficiente, o restaurante deve atentar-se para a variável preço (IGLESIAS; GUILLÉN, 2004). O preço é um critério importante para escolher um restaurante além de influenciar a satisfação (NAMIN, 2017). Entretanto, é subjetivo e depende da experiência anterior do consumidor, isso porque é a percepção do cliente em relação a justiça, racionalidade, adequação ou comparação com outros (MCNEIL; YOUNG, 2019). McNeil e Young (2019), encontraram uma influência positiva do preço, sendo uma variável sensível a variações e diferenças de valores. Dessa forma, o preço se torna um fator relativo em face da qualidade percebida pelo consumidor e a percepção de preço é baseada na comparação que o consumidor faz com os concorrentes (KAEWMAHAPHINYO, NUANGJAMNONG E DOWPISET, 2020; KIN, NG E KIM, 2009; KONUK, 2019). Por fim, são propostas as hipóteses finais do estudo:

H4a: O preço influencia positivamente a preocupação com os outros.

H4b: O preço influencia positivamente a expressão de sentimentos.

H4c: O preço influencia positivamente a ajudar o restaurante.

Como forma de melhor compreender as relações das variáveis propostas sobre o comportamento do consumidor, com o intuito de gerar estratégias mercadológicas para os restaurantes, apresenta-se na Figura 1 o modelo teórico da pesquisa adaptado de Jeong e Jang (2011). 
Figura 1 - Modelo teórico proposto

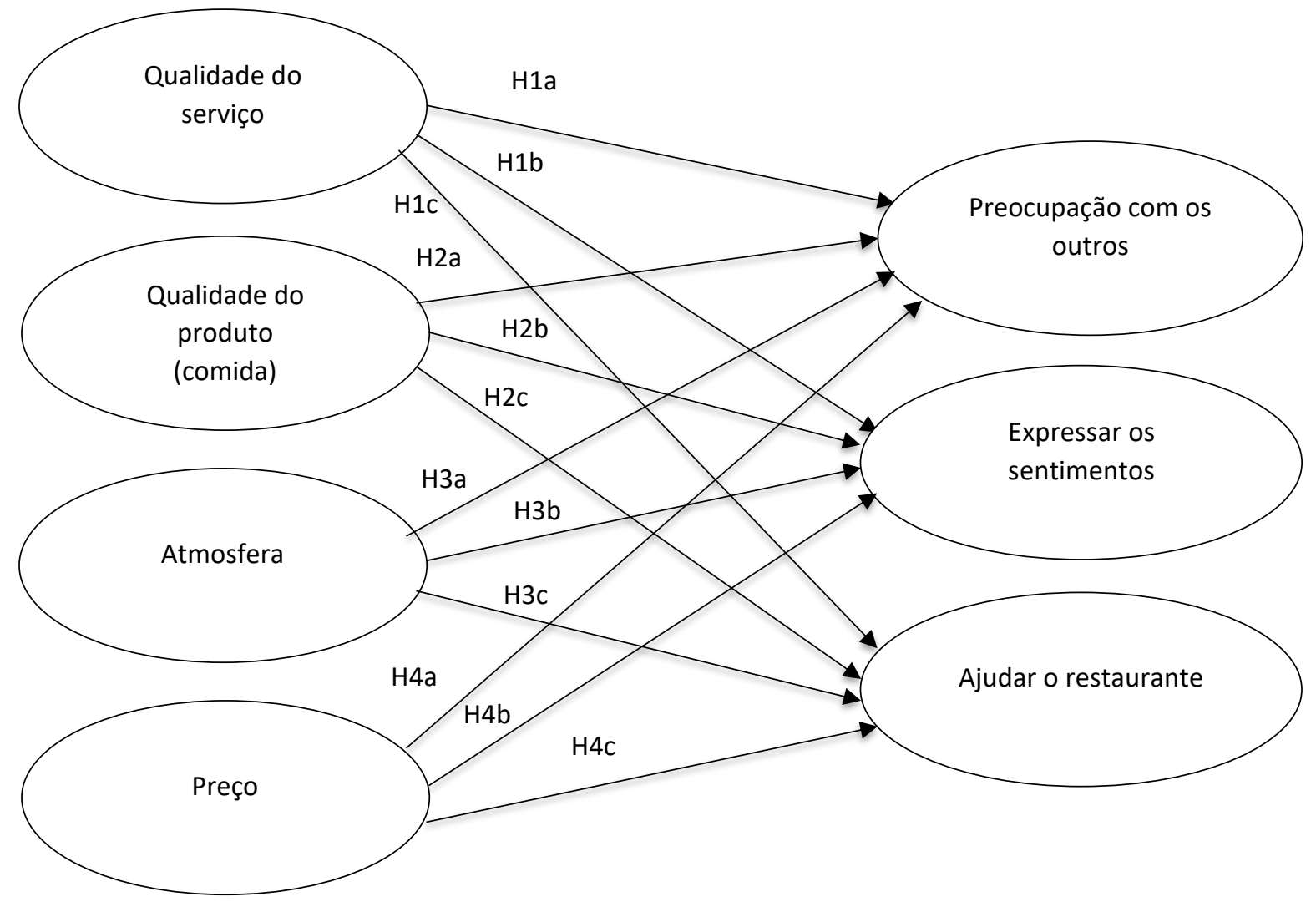

Fonte: elaborado pelos autores (2020).

\section{PROCEDIMENTOS METODOLÓGICOS}

Nesta pesquisa foi utilizada o método exploratório para identificar os fatores que influenciam o marketing boca a boca nas MPEs tendo como delineamento de pesquisa uma survey. Foi aplicado um questionário aos consumidores de restaurantes para avaliar a motivação do boca a boca por três variáveis: "preocupação com os outros", "expressão de sentimentos" e "ajuda ao restaurante". As variáveis que influenciam no âmbito das MPEs escolhidas foram: "qualidade da comida", "qualidade do serviço", a "atmosfera" e o "preço". Essas variáveis foram adaptadas da pesquisa de Jeong e Jang (2011), que teve um enfoque online, porém pequenas alterações e inclusões contribuíram para o trabalho, permitindo a análise do marketing boca a boca. Dessa forma, as contribuições de Jeong e Jang (2011) ocorreram principalmente no âmbito da determinação das variáveis.

O questionário foi aplicado numa plataforma online por meio do efeito bola de neve (ZINKHAN; BURTON; WALLENDORF, 1983), com amostra não aleatória, a partir dos contatos dos pesquisadores e obtendo inicialmente 177 respostas. Entretanto, foi inserida uma pergunta filtro com o objetivo de verificar se o consumidor realiza o marketing boca a boca no contexto de MPEs: "Você comenta com amigos, familiares ou conhecidos sobre restaurantes/lanchonetes que costuma frequentar?". E a partir de tal pergunta foram descartados 32 questionários, em que os respondentes selecionaram "não", totalizando uma amostra final de 145 respondentes. Em seguida foi perguntado entre qualidade do alimento, do serviço, da atmosfera e do preço, quais das opções o consumidor costumava enfatizar nos seus comentários, independentemente do tipo de estabelecimento frequentado. Depois foram questionados quais os tipos de restaurante/lanchonetes que frequentavam. 
Então, para que pudesse avaliar o modelo no contexto de lanchonetes classificadas como MPEs, foram criadas situações por meio da técnica projetiva (MALHOTRA, 2012). Para que o respondente conseguisse projetar o tipo de estabelecimento que estava sendo levado em consideração, foi necessário descrever características do local: "Imagine que um amigo pergunta se você tem alguma indicação de um bom lugar para comer sanduíche perto da sua casa. Seu amigo quer um estabelecimento pequeno e que seja local". Essa descrição foi criada após a realização de 15 entrevistas com consumidores em que era perguntado o que considerava em uma lanchonete classificada como MPE; após entrevistas a situação foi criada e validada por especialistas da área de marketing. E após a descrição do contexto foram criadas situações que envolviam as variáveis independentes, sendo que em cada situação apenas uma variável era alterada negativamente, e então, deveria ser escolhida qual situação faria a pessoa indicar determinado lugar.

Nas partes finais do questionário as perguntas foram encontradas originalmente no estudo de Jeong e Jang (2011) e traduzidas de acordo com o contexto. Posteriormente, foram validadas por dois especialistas da área de marketing e adaptadas ao layout do questionário final. As duas seções foram analisadas baseadas na escala Likert de cinco pontos: as motivações dos consumidores para realizarem boca a boca, com seis perguntas; e os itens que influenciariam sua indicação do restaurante/lanchonete, composta por dez perguntas. Um pré-teste foi conduzido e contribuiu para identificar melhorias na redação dos itens.

As variáveis e os respectivos indicadores estão apresentados no Quadro 2. A partir das variáveis e do modelo proposto, conforme a Figura 2, foi realizada a Modelagem de Equações Estruturais (SEM) por meio do software Smart PLS2.0, em que a estimação do modelo ocorre por meio das variâncias. Esse método e software foram os escolhidos, pela restrição do tamanho da amostra, afinal, foram analisados 145 respondentes.

Quadro 2 - Variáveis do estudo

\begin{tabular}{|c|c|c|}
\hline Variável & Notação & Indicadores \\
\hline \multirow{2}{*}{$\begin{array}{l}\text { Preocupação com os } \\
\text { outros }\end{array}$} & Preocup1 & $\begin{array}{l}\text { Eu quero ajudar os outros com a experiência positiva que tive no } \\
\text { restaurante }\end{array}$ \\
\hline & Preocup2 & $\begin{array}{l}\text { Eu quero dar aos outros a oportunidade de ter uma boa experiência em } \\
\text { um restaurante }\end{array}$ \\
\hline \multirow{2}{*}{$\begin{array}{l}\text { Expressar } \\
\text { sentimentos }\end{array}$} & ExpSent1 & $\begin{array}{l}\text { Dessa forma eu posso expressar a minha alegria sobre uma boa } \\
\text { experiência no restaurante }\end{array}$ \\
\hline & ExpSent2 & $\begin{array}{l}\text { Eu me sinto bem quando posso contar aos outros sobre uma boa } \\
\text { experiência em um restaurante }\end{array}$ \\
\hline \multirow[t]{2}{*}{ Ajudar o restaurante } & AjRest1 & $\begin{array}{l}\text { Fiquei tão satisfeito com a experiência no restaurante, que decidi ajudá- } \\
\text { lo a ser bem-sucedido. }\end{array}$ \\
\hline & AjRest2 & Na minha opinião, os bons restaurantes devem ser apoiados \\
\hline \multirow{3}{*}{$\begin{array}{l}\text { Qualidade do Produto } \\
\text { do Restaurante } \\
\text { (Comida) }\end{array}$} & QualCom1 & O restaurante serve comida saborosa \\
\hline & QualCom2 & A comida tem uma aparência agradável \\
\hline & QualCom3 & A comida é servida na temperatura adequada \\
\hline \multirow{3}{*}{ Qualidade do Serviço } & QualServ1 & Os funcionários são atenciosos \\
\hline & QualServ2 & O restaurante é confiável e consistente \\
\hline & QualServ3 & O restaurante tinha funcionários simpáticos e prestativos \\
\hline \multirow{3}{*}{ Atmosfera } & Atm1 & Os funcionários estavam limpos e bem vestidos \\
\hline & Atm2 & O ambiente de jantar estava limpo \\
\hline & Atm3 & O interior do restaurante era visualmente atraente \\
\hline Preço & Preço & O preço do restaurante era razoável \\
\hline
\end{tabular}

Fonte: elaborado pelos autores (2020). 
Mariana Torres Uchoa, Ricardo Limongi França Coelho e André Luiz Barboza da Silva

A confiabilidade da amostra foi analisada para avaliar a consistência das variáveis, traduzidas e utilizadas no instrumento de pesquisa, por meio da confiabilidade composta pelo Alfa de Cronbach. A medida retornou uma confiabilidade adequada, pois todos os valores foram maiores que 0,7 (HAIR et al., 2016), conforme a Tabela 1.

Tabela 1 - Confiabilidade dos Construtos

\begin{tabular}{lcccc}
\hline & AVE & Confiabilidade Composta & Alpha de Cronbach & Comunalidade \\
\hline Ajudar Restaurante & 0,7944 & 0,8854 & 0,7419 & 0,7944 \\
\hline Atmosfera & 0,6683 & 0,8578 & 0,7527 & 0,6683 \\
\hline ExpressarSentimentos & 0,7324 & 0,844 & 0,6663 & 0,7324 \\
\hline Preço & 1 & 1 & 1 & 1 \\
\hline PreocupOutros & 0,7864 & 0,8804 & 0,7289 & 0,7864 \\
\hline QualComida & 0,6745 & 0,8611 & 0,7628 & 0,6745 \\
\hline QualServico & 0,6412 & 0,8426 & 0,7229 & 0,6412 \\
\hline
\end{tabular}

Fonte: elaborado pelos autores (2020).

Foram analisadas também duas validações do modelo: a convergente e a discriminante. A avaliação da validade convergente foi realizada por meio das cargas fatoriais, em que todos os indicadores do modelo apresentaram cargas fatoriais maiores que 0,75 , indicando que mensuram a variável correta, conforme a Tabela 2. Além disso, a validade convergente também foi avaliada pelo AVE, apresentado na Tabela 1, em que os resultados foram superiores a 0,5, conforme indica Byrne (2016), assim, é possível concluir que os construtos, de fato, medem as variáveis.

Tabela 2 - Validade Convergente

\begin{tabular}{lccccccc}
\hline & $\begin{array}{c}\text { Ajudar } \\
\text { Restaurante }\end{array}$ & Atmosfera & $\begin{array}{c}\text { Expressar } \\
\text { Sentimentos }\end{array}$ & Preço & $\begin{array}{c}\text { Preocup } \\
\text { Outros }\end{array}$ & $\begin{array}{c}\text { Qual } \\
\text { Comida }\end{array}$ & $\begin{array}{c}\text { Qual } \\
\text { Serviço }\end{array}$ \\
\hline AjRest1 & 0,9041 & 0 & 0 & 0 & 0 & 0 & 0 \\
\hline AjRest2 & 0,8783 & 0 & 0 & 0 & 0 & 0 & 0 \\
\hline Atm1 & 0 & 0,8198 & 0 & 0 & 0 & 0 & 0 \\
\hline Atm2 & 0 & 0,8609 & 0 & 0 & 0 & 0 & 0 \\
\hline Atm3 & 0 & 0,7691 & 0 & 0 & 0 & 0 & 0 \\
\hline ExpSentime1 & 0 & 0 & 0,944 & 0 & 0 & 0 & 0 \\
\hline ExpSentime2 & 0 & 0 & 0,7574 & 0 & 0 & 0 & 0 \\
\hline Preço & 0 & 0 & 0 & 1 & 0 & 0 & 0 \\
\hline Preocup1 & 0 & 0 & 0 & 0 & 0,8759 & 0 & 0 \\
\hline Preocup2 & 0 & 0 & 0 & 0 & 0,8975 & 0 & 0 \\
\hline QualCom1 & 0 & 0 & 0 & 0 & 0 & 0,7603 & 0 \\
\hline QualComid2 & 0 & 0 & 0 & 0 & 0 & 0,8326 & 0 \\
\hline QualiCom3 & 0 & 0 & 0 & 0 & 0 & 0,8674 & 0 \\
\hline QualiSer3 & 0 & 0 & 0 & 0 & 0 & 0 & 0,7749 \\
\hline QualiServ1 & 0 & 0 & 0 & 0 & 0 & 0 & 0,851 \\
\hline Qualiser2 & 0 & 0 & 0 & 0 & 0 & 0 & 0,774 \\
\hline
\end{tabular}

Fonte: elaborado pelos autores (2020).

A validade discriminante, que visa avaliar se a variável está medindo mais de uma dimensão, foi testada por meio das cargas fatoriais cruzadas, como apresentado na Tabela 3. O resultado mostrou que as cargas dos indicadores são maiores em seus respectivos construtos do que nos outros construtos. 
Comunicação boca a boca como instrumento de marketing para micro e pequenas empresas

Tabela 3 - Validade discriminante

\begin{tabular}{llllllll}
\hline & $\begin{array}{l}\text { Ajudar } \\
\text { Restaurante }\end{array}$ & Atmosfera & $\begin{array}{l}\text { Expressar } \\
\text { Sentimentos }\end{array}$ & Preço & $\begin{array}{l}\text { Preocup } \\
\text { Outros }\end{array}$ & $\begin{array}{l}\text { Qual } \\
\text { Comida }\end{array}$ & $\begin{array}{l}\text { Qual } \\
\text { Serviço }\end{array}$ \\
\hline AjRest1 & $\mathbf{0 , 9 0 4 1}$ & 0,2063 & 0,3545 & 0,0297 & 0,2775 & 0,0453 & 0,2824 \\
\hline AjRest2 & $\mathbf{0 , 8 7 8 3}$ & 0,2069 & 0,2738 & $-0,0617$ & 0,2615 & 0,0865 & 0,2184 \\
\hline Atm1 & 0,1574 & $\mathbf{0 , 8 1 9 8}$ & 0,1834 & 0,1218 & 0,2448 & 0,5416 & 0,6719 \\
\hline Atm2 & 0,2287 & $\mathbf{0 , 8 6 0 9}$ & 0,1945 & 0,2666 & 0,3157 & 0,5352 & 0,5036 \\
\hline Atm3 & 0,1751 & $\mathbf{0 , 7 6 9 1}$ & 0,3425 & 0,4206 & 0,2553 & 0,397 & 0,5586 \\
\hline ExpSentimentos1 & 0,2938 & 0,3121 & $\mathbf{0 , 9 4 4}$ & 0,2061 & 0,4017 & 0,2007 & 0,3721 \\
\hline ExpSentimentos2 & 0,3501 & 0,1746 & $\mathbf{0 , 7 5 7 4}$ & 0,0652 & 0,2601 & 0,0816 & 0,1887 \\
\hline Preço & $-0,0151$ & 0,347 & 0,1802 & $\mathbf{1}$ & 0,1865 & 0,2627 & 0,3185 \\
\hline Preocup1 & 0,2692 & 0,2607 & 0,261 & 0,2393 & $\mathbf{0 , 8 7 5 9}$ & 0,1243 & 0,1835 \\
\hline Preocup2 & 0,2679 & 0,3313 & 0,4438 & 0,0981 & $\mathbf{0 , 8 9 7 5}$ & 0,1783 & 0,2119 \\
\hline QualCom1 & 0,0484 & 0,3547 & 0,0929 & 0,1532 & 0,1156 & $\mathbf{0 , 7 6 0 3}$ & 0,3069 \\
\hline QualComid2 & 0,0244 & 0,5585 & 0,1868 & 0,2199 & 0,132 & $\mathbf{0 , 8 3 2 6}$ & 0,4571 \\
\hline QualiCom3 & 0,0999 & 0,5217 & 0,155 & 0,2559 & 0,1686 & $\mathbf{0 , 8 6 7 4}$ & 0,3949 \\
\hline QualiSer3 & 0,1834 & 0,6025 & 0,24 & 0,246 & 0,1998 & 0,3774 & $\mathbf{0 , 7 7 4 9}$ \\
\hline QualiServ1 & 0,2766 & 0,4936 & 0,3405 & 0,2094 & 0,1917 & 0,3792 & $\mathbf{0 , 8 5 1}$ \\
\hline Qualiser2 & 0,2065 & 0,6192 & 0,2512 & 0,3304 & 0,1443 & 0,3983 & $\mathbf{0 , 7 7 4}$ \\
\hline
\end{tabular}

Fonte: elaborado pelos autores (2020).

A validade discriminante foi mensurada também por meio do critério de Fornell e Larcker (1981), que não apresentou problemas, como apresentado na Tabela 4.

Tabela 4 - Validade discriminante

\begin{tabular}{lccccccc}
\hline & $\begin{array}{c}\text { Ajudar } \\
\text { Restaurante }\end{array}$ & $\begin{array}{c}\text { Atmosfer } \\
\mathbf{a}\end{array}$ & $\begin{array}{c}\text { Expressar } \\
\text { Sentimentos }\end{array}$ & Preço & $\begin{array}{c}\text { Preocup } \\
\text { Outros }\end{array}$ & $\begin{array}{c}\text { Qual } \\
\text { Comida }\end{array}$ & $\begin{array}{c}\text { Qual } \\
\text { Serviço }\end{array}$ \\
\hline Ajudar Restaurante & $\mathbf{0 , 8 9 1}$ & 0,000 & 0,000 & 0,000 & 0,000 & 0,000 & 0,000 \\
\hline Atmosfera & 0,232 & $\mathbf{0 , 8 1 7}$ & 0,000 & 0,000 & 0,000 & 0,000 & 0,000 \\
\hline ExpressarSentimentos & 0,355 & 0,302 & $\mathbf{0 , 8 5 6}$ & 0,000 & 0,000 & 0,000 & 0,000 \\
\hline Preço & $-0,015$ & 0,347 & 0,180 & $\mathbf{1 , 0 0 0}$ & 0,000 & 0,000 & 0,000 \\
\hline PreocupOutros & 0,303 & 0,335 & 0,402 & 0,187 & $\mathbf{0 , 8 8 7}$ & 0,000 & 0,000 \\
\hline QualComida & 0,073 & 0,596 & 0,182 & 0,263 & 0,172 & $\mathbf{0 , 8 2 1}$ & 0,000 \\
\hline QualServico & 0,283 & 0,700 & 0,352 & 0,319 & 0,224 & 0,477 & $\mathbf{0 , 8 0 1}$ \\
\hline
\end{tabular}

Fonte: elaborado pelos autores (2020).

\section{ANÁLISE DOS RESULTADOS}

A amostra foi composta por $55 \%$ dos respondentes do gênero feminino, $75 \%$ com até 24 anos, e $36 \%$ com a faixa de renda entre $R \$ 6.780$ e $R \$ 13.560,00.66 \%$ possuem ensino superior incompleto e $40 \%$ se alimentam fora de casa de quatro a sete vezes por semana.

\subsection{MODELO ESTRUTURAL}

O modelo estrutural proposto foi estimado por modelagem de equações estruturais no software PLS, com estimação das variâncias para avaliar a relação entre a experiência do consumidor no restaurante e sua motivação para realizar o boca a boca. Após a análise de ajuste do modelo, realizado por meio da confiabilidade, validade convergente e validade discriminante, foram avaliadas as relações individuais entre os construtos. Os resultados do modelo são apresentados na Tabela 5 e, após os testes, das relações observadas, três foram consideradas estatisticamente significativas: 
Mariana Torres Uchoa, Ricardo Limongi França Coelho e André Luiz Barboza da Silva

Tabela 5 - Resultado Modelo Estrutural

\begin{tabular}{lcccc}
\hline \multicolumn{1}{c}{ Relação entre os construtos } & $\begin{array}{c}\text { Sample } \\
\text { Mean (M) }\end{array}$ & $\begin{array}{c}\text { Standard Deviation } \\
\text { (STDEV) }\end{array}$ & $\begin{array}{c}\text { T Statistics } \\
\text { (|O/STERR|) }\end{array}$ & Pvalor \\
\hline QualServico -> PreocupOutros & $-0,0207$ & 0,1316 & 0,221 & 0,825 \\
\hline QualServico -> ExpressarSentimentos & 0,2632 & 0,147 & 1,8381 & $0,068^{*}$ \\
\hline QualServico -> Ajudar Restaurante & 0,2618 & 0,1353 & 1,9857 & $0,049^{* *}$ \\
\hline QualComida -> PreocupOutros & $-0,016$ & 0,1319 & 0,3641 & 0,716 \\
\hline QualComida -> ExpressarSentimentos & 0,0227 & 0,1217 & 0,2206 & 0,826 \\
\hline QualComida -> Ajudar Restaurante & $-0,0821$ & 0,1385 & 0,8357 & 0,405 \\
\hline Atmosfera -> PreocupOutros & 0,338 & 0,1527 & 2,323 & $0,022^{* *}$ \\
\hline Atmosfera -> ExpressarSentimentos & 0,1018 & 0,1494 & 0,7112 & 0,478 \\
\hline Atmosfera -> Ajudar Restaurante & 0,1596 & 0,1369 & 1,1365 & 0,258 \\
\hline Preço -> PreocupOutros & 0,09 & 0,1264 & 0,6751 & 0,501 \\
\hline Preço -> ExpressarSentimentos & 0,0551 & 0,0951 & 0,676 & 0,500 \\
\hline Preço -> Ajudar Restaurante & $-0,1314$ & 0,0923 & 1,3452 & 0,181 \\
\hline
\end{tabular}

Fonte: elaborado pelos autores (2020).

A "atmosfera" teve uma influência significante e positiva, com $95 \%$ de confiança, na motivação de realizar o boca a boca por "preocupação com os outros" (stdev =0,11527). A "qualidade do serviço" teve uma influência significante e positiva em duas variáveis, "ajudar o restaurante", com 95\% de confiança (stedv =0,1353), e "expressar os sentimentos" com $90 \%$ de confiança (stdev $=0,147$ ), como motivação para realizar o boca a boca. conforme apresentado na Figura 2

Figura 2 - Resultado Modelo estrutural

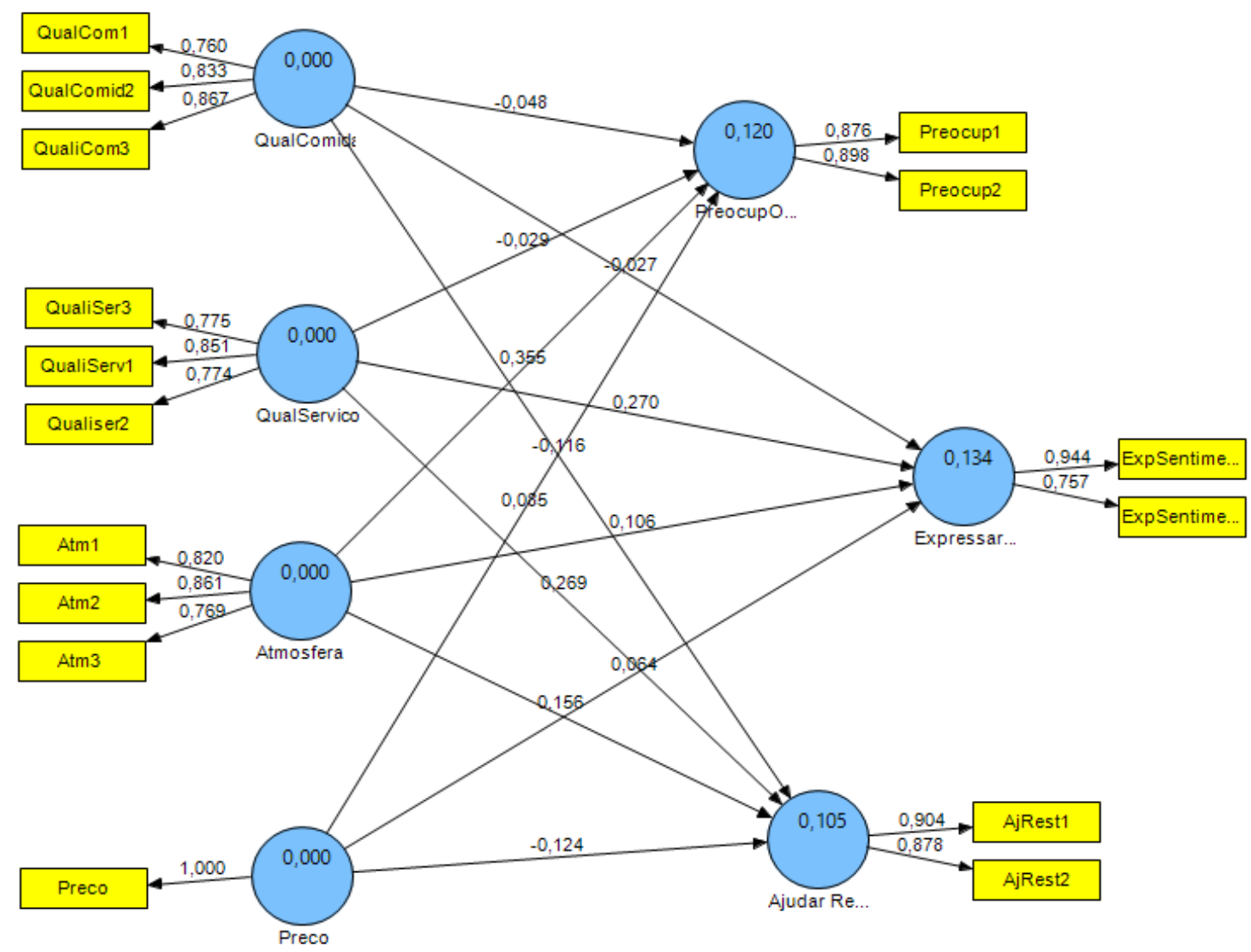

Fonte: elaborado pelos autores (2020).

Dessa forma percebe-se que a "qualidade do serviço" atua como antecedente do marketing boca a boca. Os outros resultados indicam que o "preço" e a "qualidade da comida" 
não possuem influência significativa para que um consumidor realize o boca a boca em relação a nenhuma das três motivações. A "atmosfera" não tem relação significativa em "ajudar o restaurante" ou "para expressar os sentimentos". E a "qualidade do serviço" não é significativa, ou seja, não é suficiente para que um consumidor faça um boca a boca por "preocupação com os outros". Por fim, é possível avaliar que as motivações dos consumidores para realizar o boca a boca em MPE são influenciadas por aspectos de "qualidade do serviço" e "atmosfera", entretanto a "qualidade da comida" e o "preço" não são relevantes para a divulgação e comentário do restaurante/lanchonete.

Quadro 3-Resultado das Hipóteses
\begin{tabular}{|c|l|c|c|}
\hline Hipótese & \multicolumn{1}{|c|}{ Relação entre os construtos } & Relação & Resultado \\
\hline H1a & QualServico -> PreocupOutros & $--->+$ & Não suportada \\
\hline H1b & QualServico -> ExpressarSentimentos & $--->+$ & Suportada \\
\hline H1c & QualServico -> Ajudar Restaurante & $--->+$ & Suportada \\
\hline H2a & QualComida -> PreocupOutros & $--->+$ & Não suportada \\
\hline H2b & QualComida -> ExpressarSentimentos & $--->+$ & Não suportada \\
\hline H2c & QualComida -> Ajudar Restaurante & $--->+$ & Não suportada \\
\hline H3a & Atmosfera -> PreocupOutros & $--->+$ & Suportada \\
\hline H3b & Atmosfera -> ExpressarSentimentos & $--->+$ & Não suportada \\
\hline H3c & Atmosfera -> Ajudar Restaurante & $--->+$ & Não suportada \\
\hline H4a & Preço -> PreocupOutros & $--->+$ & Não suportada \\
\hline H4b & Preço -> ExpressarSentimentos & $--->+$ & Não suportada \\
\hline H4c & Preço -> Ajudar Restaurante & $--->+$ & Não suportada \\
\hline
\end{tabular}

Fonte: elaborado pelos autores (2020).

\subsection{DISCUSSÃO DOS RESULTADOS}

Os resultados encontrados no modelo indicam que para realizar o boca a boca com o intuito de "ajudar o restaurante" e "expressar os sentimentos", a "qualidade do serviço" é um fator determinante, corroborando o estudo de Jeong e Jang (2011). Outros estudos (ex.: Iglesias e Guillén, 2004; Canny, 2013; Canny, 2014) concluíram que a qualidade do serviço é a variável mais importante para a satisfação do cliente, ressaltando que no contexto de MPE(s) a expectativa dos consumidores é por um atendimento memorável, que poderia, por exemplo, ser superior à qualidade do produto.

Neste sentido, apesar da literatura indicar que a qualidade da comida é um componente fundamental da experiência do restaurante (NAMKUNG; JANG, 2007), que influencia a fidelidade do cliente na escolha do restaurante (CLARK; KAMINSKI; RINK, 1992) e que está entre os elementos mais importantes na satisfação do cliente no segmento de restaurantes (LAW; HUI; ZHAO, 2004), este estudo encontrou, no contexto analisado, um resultado diferente, ou seja, a variável "qualidade da comida" não teve nenhuma influência significativa nas motivações estudadas. Em comparação com os estudos de Jeong e Jang (2011) a única diferença é que no estudo a "comida" teve influência significativa em "ajudar o restaurante" e as outras variáveis não foram estatisticamente significativas. Além disso, a "atmosfera" também teve uma influência positiva apenas na "preocupação com os outros", porém não foram significativas em "ajudar o restaurante" e para "expressar os sentimentos", o que também está alinhado com Jeong e Jang (2011) e Canny (2014). Logo, é por meio da atmosfera que os consumidores experimentam novas sensações em ambientes casuais e descontraídos, por meio da decoração, iluminação, cores e layout (CANNY, 2013; CANNY, 
2014). O preço não teve influência significativa nas motivações estudadas, assim como apontado nos estudos de Kin, Ng e Kim (2009) e Jeong e Jang (2011) e, que argumentam que o preço é um fator relativo. Porém, estudos como de Jin e Lee (2016) e Namin (2017) encontraram que o preço é um critério significativo para influenciar no boca a boca.

Desta forma, é importante que os restaurantes/lanchonetes entendam quais as principais motivações que seus consumidores possuem para satisfazer suas necessidades, e então, alcançarem resultados melhores nas ações de divulgação, assim como o boca a boca. Afinal, as empresas precisam garantir a satisfação e a confiança dos consumidores já que essas variáveis têm uma associação positiva com a retenção dos consumidores e o marketing boca a boca no contexto das MPEs (Ranaweera e Prabhu, 2003).

\section{CONSIDERAÇÕES FINAIS}

Esta pesquisa teve como objetivo analisar os fatores que influenciam o consumidor a indicar uma MPE por meio do boca a boca. Com a revisão teórica e a análise de dados foi verificado que a divulgação de uma MPE no segmento de alimentação, é influenciado pelas variáveis "qualidade do serviço" (que inclui atendimento, apresentação dos funcionários e confiabilidade do restaurante) e atmosfera. É compreendido então que quando o consumidor pensa no seu bem-estar e no que realmente agrada, o importante é o serviço prestado: funcionários simpáticos, bem-humorados, que atendem bem, fazem a pessoa se sentir à vontade.

Já o lugar, como o ambiente familiar, aconchego, ou conforto são aspectos importantes quando o consumidor indica um restaurante com o objetivo de preocupação com os outros. Percebe-se, então, que quando o consumidor quer agradar, ou seja, quando pensa no bem-estar de outras pessoas os itens como ambiente, limpeza e design do lugar são avaliados. Essa preocupação com a atmosfera ocorre porque, diante das situações expostas no questionário que enfatizavam e destacavam as características do local que era pesquisado, existem pessoas que não frequentam lugares mais simples com cadeiras de plástico, mesas na calçada, uma realidade frequente nas MPEs. Além disso, a qualidade do alimento e o preço são fatores menos relevantes em uma motivação, ou seja, não alteraram a realização do boca a boca. O preço dos alimentos não trouxe um resultado significativo, pois normalmente as pessoas ao frequentarem um restaurante ou lanchonete pesquisam antes sobre o local (internet ou boca a boca), dessa forma já possuem uma noção sobre como funciona o estabelecimento. Entretanto, caso o preço seja uma surpresa para o consumidor que não obteve nenhuma informação anterior, ao chegar no restaurante e olhar o cardápio é possível identificar se esse ponto atende ou não as expectativas e continuar com o atendimento ou desistir antes do consumo.

Dessa forma, o estudo apresenta duas implicações. A primeira é que os donos dos restaurantes e lanchonetes de MPEs devem investir principalmente na qualidade do serviço prestado ao consumidor, pois um atendimento satisfatório prestado com qualidade resultará em uma satisfatória indicação. Em contrapartida, a pesquisa mostra que o consumidor ao valorizar essa variável, destaca que o atendimento, em geral, é uma deficiência nos restaurantes/lanchonetes de maneira geral. A segunda implicação tange o preço e qualidade do alimento, os resultados mostram que se o consumidor frequenta o restaurante é porque está satisfeito com o produto e a variação do preço não é motivo para que as pessoas deixem de indicá-lo, desde que as necessidades como consumidor estejam sendo atendidas.

A primeira limitação do estudo diz respeito ao contexto, setor de serviços, talvez um estudo focado na análise da aquisição de um produto resultasse em diferentes variáveis de 
influência da pesquisa atual. A segunda limitação está nas motivações analisadas, seria relevante avaliar outras motivações que os consumidores possuem para divulgarem as empresas (descontos com indicação, etc.) e quais itens, por exemplo, do composto mercadológico influenciam o marketing boca a boca de maneira mais significativa (localização, combos - parecidos com grandes redes).

Como sugestão de continuidade em estudos futuros, um aspecto seria analisar junto aos gestores das MPEs, quais fatores são os mais valorizados por eles, e avaliar se as empresas estão alinhadas com as expectativas dos consumidores. Poderia também ser pesquisada a diferença e o impacto que tem o boca a boca em franquias, alinhando a estratégia de marketing de uma rede em relação ao atendimento em diferentes regiões. $E$, por fim, outra sugestão é a análise do ambiente online para o boca a boca e seu impacto no desempenho da empresa.

\section{REFERÊNCIAS}

ABRASEL. Brasileiro aumenta despesa com alimentação fora de casa [Web Site]. Disponível em: https://abrasel.com.br/noticias/noticias/brasileiro-aumenta-despesa-com-alimentacaofora-de-casa/. Acesso em: 02 abr. 2021

ABRASEL. Bobs é destaque no mercado por pioneirismo [Web Site]. Disponível em: https://abrasel.com.br/noticias/noticias/bobs-e-destaque-no-mercado-por-pioneirismo/. Acesso em: 02 abr. 2021.

ARNDT, J. Role of product-related conversations in the diffusion of a new product. Journal of Marketing Research, v. 4, n. 3, p. 291-295, 1967. DOI:

https://doi.org/10.1177/002224376700400308.

BAHAUDDIN, A. R.; WEI, L. S.; MANTIHAL, S. Food Sensory Factors and Restaurant Images on Customer Satisfaction: A Comparison of Franchise and Local Fast-Food Restaurant. Asian Journal of Entrepreneurship, v. 1, n. 4, p. 62-71, 2020.

BAKER, D. A.; CROMPTON, J. L. Quality, satisfaction and behavioral intentions. Annals of Tourism research, v. 27, n. 3, p. 785-804, 2000. DOI: https://doi.org/10.1016/S01607383(99)00108-5.

BAMBAUER-SACHSE, S.; MANGOLD, S. Brand equity dilution through negative online wordof-mouth communication. Journal of Retailing and Consumer Services, v. 18, n. 1, p. 38-45, 2011. DOI: https://doi.org/10.1016/j.jretconser.2010.09.003.

BROWN, T. J.; BARRY, T. E.; DACIN, P. A.; GUNST, R. F. Spreading the word: Investigating antecedents of consumers' positive word-of-mouth intentions and behaviors in a retailing context. Journal of the Academy of Marketing Science, v. 33, n. 2, p. 123-138, 2005. DOI: https://doi.org/10.1177/0092070304268417.

BYRNE, B. M. Structural equation modeling with AMOS: Basic concepts, applications, and programming. 2016.

CANNY, I. U. The role of food quality, service quality, and physical environment on customer satisfaction and future behavioral intentions in casual dining restaurant. In: The 7th National 
Mariana Torres Uchoa, Ricardo Limongi França Coelho e André Luiz Barboza da Silva

Research Management Conference, Sriwijaya University-Palembang, Indonesia (27-28 November 2013). 2013. DOI: http://dx.doi.org/10.2139/ssrn.2363339.

CANNY, I. U. Measuring the mediating role of dining experience attributes on customer satisfaction and its impact on behavioral intentions of casual dining restaurant in Jakarta. International Journal of Innovation, Management and Technology, v. 5, n. 1, p. 2529, 2014. DOI: http://dx.doi.org/10.7763/IJIMT.2014.V5.480.

CASIDY, R.; SHIN, H. The effects of harm directions and service recovery strategies on customer forgiveness and negative word-of-mouth intentions. Journal of Retailing and Consumer Services, v. 27, p. 103-112, 2015. DOI:

https://doi.org/10.1016/j.jretconser.2015.07.012

CHEVALIER, J. A.; MAYZLIN, D. The effect of word of mouth on sales: Online book reviews. Journal of Marketing Research, v. 43, n. 3, p. 345-354, 2006. DOI: https://doi.org/10.1509/jmkr.43.3.345.

CLARK, G. L.; KAMINSKI, P. F.; RINK, D. R. Consumer complaints: Advice on how companies should respond based on an empirical study. Journal of Consumer Marketing, 1992. DOI: https://doi.org/10.1108/07363769210035189

COLLIER, J. E.; BIENSTOCK, C. C. Measuring service quality in e-retailing. Journal of Service Research, v. 8, n. 3, p. 260-275, 2006. DOI: https://doi.org/10.1177/1094670505278867

EAST, R.; HAMMOND, K.; LOMAX, W. Measuring the impact of positive and negative word of mouth on brand purchase probability. International Journal of Research in Marketing, v. 25, n. 3, p. 215-224, 2008. DOI: https://doi.org/10.1016/j.ijresmar.2008.04.001.

HA, J.; JANG, S. S. Effects of service quality and food quality: The moderating role of atmospherics in an ethnic restaurant segment. International Journal of Hospitality Management, v. 29, n. 3, p. 520-529, 2010. DOI: https://doi.org/10.1016/j.ijhm.2009.12.005.

HAIR JR, J. F.; HULT, G. T. M.; RINGLE, C. M.; SARSTEDT, M. A primer on partial least squares structural equation modeling (PLS-SEM). Sage publications, 2016.

HARRISON-WALKER, L. J. The measurement of word-of-mouth communication and an investigation of service quality and customer commitment as potential antecedents. Journal of Service Research, v. 4, n. 1, p. 60-75, 2001. DOI:

https://doi.org/10.1177/109467050141006

HEUNG, V. CS; GU, T. Influence of restaurant atmospherics on patron satisfaction and behavioral intentions. International Journal of Hospitality Management, v. 31, n. 4, p. 11671177, 2012. https://doi.org/10.1016/j.ijhm.2012.02.004

IFIE, K. SIMINTIRAS, A. C.; DWIVEDI, Y.; MAVRIDOU, V. How service quality and outcome confidence drive pre-outcome word-of-mouth. Journal of Retailing and Consumer Services, v. 44, p. 214-221, 2018. DOI: https://doi.org/10.1016/j.jretconser.2018.07.002.

IGLESIAS, M. P.; GUILLÉN, M. J. Y. Perceived quality and price: their impact on the 
satisfaction of restaurant customers. International Journal of Contemporary Hospitality Management, v. 16, n. 6, p. 373-379, 2004. DOI:

https://doi.org/10.1108/09596110410550824.

JEONG, E.; JANG, S. S. Restaurant experiences triggering positive electronic word-of-mouth (eWOM) motivations. International Journal of Hospitality Management, v. 30, n. 2, p. 356366, 2011. DOI: https://doi.org/10.1016/j.ijhm.2010.08.005.

JIN, N.; LEE, S. The impact of restaurant experiences on mature and nonmature customers: exploring similarities and differences. International Journal of Hospitality \& Tourism Administration, v. 17, n. 1, p. 1-26, 2016. DOI: https://doi.org/10.1080/15256480.2016.1123580.

JIN, N.; MERKEBU, J.; LINE, N. D. The examination of the relationship between experiential value and price fairness in consumers' dining experience. Journal of Foodservice Business Research, v. 22, n. 2, p. 150-166, 2019. DOI: https://doi.org/10.1080/15378020.2019.1592652.

JUNG, N. Y.; SEOCK, Y. Effect of service recovery on customers' perceived justice, satisfaction, and word-of-mouth intentions on online shopping websites. Journal of Retailing and Consumer Services, v. 37, p. 23-30, 2017. DOI:

https://doi.org/10.1016/j.jretconser.2017.01.012.

KAEWMAHAPHINYO, T.; NUANGJAMNONG, C.; DOWPISET, K. Factors Influencing Customer Satisfaction and Behavioral Intention for Fast-Casual Restaurants (A Case Study in a Shopping Center, Bangkok). Available at SSRN, 2020. DOI: http://dx.doi.org/10.2139/ssrn.3787925.

KAU, A.; LOH, E. W. The effects of service recovery on consumer satisfaction: a comparison between complainants and non-complainants. Journal of Services Marketing, 2006. DOI: https://doi.org/10.1108/08876040610657039.

KIM, W. G.; NG, C. Y. N.; KIM, Y. Influence of institutional DINESERV on customer satisfaction, return intention, and word-of-mouth. International Journal of Hospitality Management, v. 28, n. 1, p. 10-17, 2009. DOI: https://doi.org/10.1016/j.ijhm.2008.03.005.

KONUK, F. The influence of perceived food quality, price fairness, perceived value and satisfaction on customers' revisit and word-of-mouth intentions towards organic food restaurants. Journal of Retailing and Consumer Services, v. 50, p. 103-110, 2019. DOI: https://doi.org/10.1016/j.jretconser.2019.05.005.

LAW, A. K.Y; HUI, Y. V.; ZHAO, X. Modeling repurchase frequency and customer satisfaction for fast food outlets. International Journal of Quality \& Reliability Management, 2004. DOI: https://doi.org/10.1108/02656710410536563.

LOUREIRO, S. M. C.; CAVALLERO, L.; MIRANDA, F. J. Fashion brands on retail websites: Customer performance expectancy and e-word-of-mouth. Journal of Retailing and Consumer Services, v. 41, p. 131-141, 2018. DOI:

https://doi.org/10.1016/j.jretconser.2017.12.005. 
Mariana Torres Uchoa, Ricardo Limongi França Coelho e André Luiz Barboza da Silva

MATHAYOMCHAN, B.; TAECHARUNGROJ, V. "How was your meal?" Examining customer experience using Google maps reviews. International Journal of Hospitality Management, v. 90, p. 102641, 2020. DOI: https://doi.org/10.1016/j.ijhm.2020.102641.

MCNEIL, P.; YOUNG, C. A. Customer satisfaction in gourmet food trucks: Exploring attributes and their relationship with customer satisfaction. Journal of Foodservice Business Research, v. 22, n. 4, p. 326-350, 2019. DOI: https://doi.org/10.1080/15378020.2019.1614400.

MILLER, J. L.; CRAIGHEAD, C. W.; KARWAN, K. R. Service recovery: a framework and empirical investigation. Journal of Operations Management, v. 18, n. 4, p. 387-400, 2000. DOI: https://doi.org/10.1016/S0272-6963(00)00032-2.

MITCHELL, R.; HUTCHINSON, K.; QUINN, B.; GILMORE, A. A framework for SME retail branding. Journal of Marketing Management, v. 31, n. 17-18, p. 1818-1850, 2015. DOI: https://doi.org/10.1080/0267257X.2015.1063531.

NAMIN, A. Revisiting customers' perception of service quality in fast food restaurants. Journal of Retailing and Consumer Services, v. 34, p. 70-81, 2017. DOI: https://doi.org/10.1016/j.jretconser.2016.09.008.

NAMKUNG, Y.; JANG, S. Does food quality really matter in restaurants? Its impact on customer satisfaction and behavioral intentions. Journal of Hospitality \& Tourism Research, v. 31, n. 3, p. 387-409, 2007. DOI: https://doi.org/10.1177/1096348007299924.

NOONE, B. M. Overcompensating for severe service failure: perceived fairness and effect on negative word-of-mouth intent. Journal of Services Marketing, v. 26, n. 5, p. 342-351, 2012. DOI: https://doi.org/10.1108/08876041211245254.

OMAR, O.; FRASER, P. SME retailing in the UK. Entrepreneurship marketing: Principles and practice of SME marketing, p. 211-229, 2010.

PEZENKA, I.; WEISMAYER, C. Which factors influence locals' and visitors' overall restaurant evaluations?. International Journal of Contemporary Hospitality Management, v. 32, n. 9, p. 2793-2812, 2020. DOI: https://doi.org/10.1108/IJCHM-09-2019-0796.

RANAWEERA, C.; PRABHU, J. On the relative importance of customer satisfaction and trust as determinants of customer retention and positive word of mouth. Journal of Targeting, Measurement and Analysis for marketing, v. 12, n. 1, p. 82-90, 2003. DOI: https://doi.org/10.1057/palgrave.jt.5740100.

RELLING, M.; SCHNITTKA, O.; SATTLER, H.; JOHNEN, M. Each can help or hurt: Negative and positive word of mouth in social network brand communities. International Journal of Research in Marketing, v. 33, n. 1, p. 42-58, 2016. DOI: https://doi.org/10.1016/j.ijresmar.2015.11.001.

SEBRAE. Micro e pequenas empresas geram 27\% do pib do Brasil [Web Page] Disponível em: https://m.sebrae.com.br/sites/PortalSebrae/ufs/mt/noticias/micro-e-pequenasempresas-geram-27-do-pib-do-brasil,ad0fc70646467410VgnVCM2000003c74010aRCRD. Acesso em: 02 maio 2020. 
SEBRAE. O perfil das Microempresas e Empresas de Pequeno Porte. Disponível em: https://www.sebrae.com.br/Sebrae/Portal\%20Sebrae/UFs/RO/Anexos/Perfil\%20das\%20ME \%20e\%20EPP\%20-\%2004\%202018.pdf. Acesso em: 02 abr. 2021.

SOLUNOĞLU, A. Perceived Food Quality, Satisfaction, Brand Image In Restaurants And Recommendation Intention Relation. Journal of Yaşar University, v. 15, n. 60, p. 833-849. 2020. DOI: https://doi.org/10.19168/jyasar.716064.

STOKES, D. Putting entrepreneurship into marketing: the processes of entrepreneurial marketing. Journal of Research in Marketing and Entrepreneurship, v. 2, n. 1, p. 1-16, 2000. DOI: https://doi.org/10.1108/14715200080001536.

SUSSKIND, A. M. I told you so! Restaurant customers' word-of-mouth communication patterns. Cornell Hotel and Restaurant Administration Quarterly, v. 43, n. 2, p. 75-85, 2002. DOI: https://doi.org/10.1016/S0010-8804(02)80034-9.

USLU, A. The relationship of service quality dimensions of restaurant enterprises with satisfaction, behavioral intention, eWOM and the moderator effect of atmosphere. Tourism \& Management Studies, v. 16, n. 3, p. 23-35, 2020. DOI: https://doi.org/10.18089/tms.2020.160303.

ZHANG, L.; MA, B.; CARTWRIGHT, D. K. The impact of online user reviews on cameras sales. European Journal of Marketing, v. 47, n. 7, p. 1115-1128, 2013. DOI: https://doi.org/10.1108/03090561311324237.

ZHANG, S.; LI, Y.; LIU, C.; RUAN, W. Reconstruction of the relationship between traditional and emerging restaurant brand and customer WOM. International Journal of Hospitality Management, v. 94, p. 102879, 2021. DOI: https://doi.org/10.1016/j.ijhm.2021.102879.

ZEITHAML, V. A.; BERRY, L. L.; PARASURAMAN, A. The nature and determinants of customer expectations of service. Journal of the academy of Marketing Science, v. 21, n. 1, p. 1-12, 1993. DOI: https://doi.org/10.1177/0092070393211001.

ZINKHAN, G. M.; BURTON, S.; WALLENDORF, M. Marketing applications for snowball sampling: Potential benefits and problems. Research methods and causal modeling in marketing, v. 1, p. 5-8, 1983.

ŽIVADINOVIĆ, B. Tourist satisfaction with quality of service, food, atmosphere, and value for money in restaurants of major cities of the Western Balkans. The European Journal of Applied Economics, v. 17, n. 2, p. 19-33, 2020. DOI: https://doi.org/10.5937/EJAE17-27360. 